Toronto, Ontario, Canada; ${ }^{2}$ Department of Community Medicine, United Arab Emirates University, Al-Ain, United Arab Emirates; ${ }^{3}$ Department of Public Health, Erasmus Medical Center, Rotterdam, The Netherlands

Introduction Infection with $M$ tuberculosis is a necessary but not sufficient condition for development of clinical Tuberculosis (TB). The reasons why some tuberculosis infections progress to clinical $\mathrm{TB}$ while most remain latent is not clear. A vegetarian diet has been implicated as a risk factor for tuberculosis among South-Asians in the UK.

Methods To explore whether this is the case in India we analysed data from the nationally representative National Family Health Survey-3 (2006) which collected information on TB and diet, and tested for HIV-1. Tuberculosis was reported by heads of households. Results Vegetarianism was not a risk factor for tuberculosis among HIV-1 negative married men and women between 15 and 49 (women) or 54 (men) years [OR 0.66 (95\% CI 0.49 to 0.89)] while poverty and a history of blood transfusions were. Individuals reporting TB were slightly older than those who did not (31.8 vs 29.4 in women, 39.3 vs 37.2 in men). Vegetarians were also slightly older than non-vegetarians (29.5 vs 29.4 in women, and 37.9 vs 37.0 in men). Except for 11 men and 11 women all individuals with TB were reported to have received treatment for their condition. Urban residence, poverty, higher age, and blood transfusion were positively associated with TB

Conclusion Vegetarianism appeared to be protective against TB, perhaps due to confounding by unmeasured life-style factors. As it seems unlikely that confounding has masked a strong positive association between vegetarianism and $\mathrm{TB}$, we conclude that vegetarianism is not a risk factor for TB in India.

\section{SP3-65 MISSION IMPOSSIBLE? EVALUATING THE EFFECTIVENESS OF HIGHLY RADICAL CANCER SURGERY WITH INSIGHTS FROM A PROSPECTIVE STUDY OF PELVIC EXENTERATION}

doi:10.1136/jech.2011.1429760.65

1,2 J Young, ${ }^{*} 1,2 \mathrm{~L}$ Rodwell, ${ }^{3} \mathrm{~A}$ Heriot, ${ }^{1,2} \mathrm{M}$ Solomon. ${ }^{1}$ University of Sydney, Sydney, New South Wales, Australia; ${ }^{2}$ Sydney Local Health Network, Sydney, New South Wales, Australia; ${ }^{3}$ Peter MacCallum Cancer Centre, Melbourne, Victoria, Australia

Introduction Policy makers, clinicians and patients need robust evidence of the effectiveness of healthcare interventions to inform decision-making. Highly aggressive cancer surgery has major implications for patients and requires substantial healthcare resources, but presents considerable challenges for evaluation. Although a randomised controlled trial is the optimal study design to investigate therapeutic effectiveness, randomisation is not possible where there are major, irreversible, differences in the treatments in intervention and control groups. Furthermore, with novel surgical techniques, as experience is gained there may be changes in patient selection, referral patterns and outcomes over time, introducing additional potential sources of bias. These methodological issues will be explored using data from a ongoing prospective, multi-centre evaluation of pelvic exenteration, a highly radical procedure for pelvic cancer where the alternative is palliative care.

Methods Baseline demographic, clinical and quality of life data are collected during the period of treatment decision-making, with clinical and quality of life outcomes assessed every 3 months thereafter. Trends in referral patterns, treatment choices, patient characteristics and outcomes will be compared between groups.

Results Since 2008, 144 patients have been assessed, with 111 (77\%) undergoing pelvic exenteration. Although the absolute rate of patient referral has increased over time, there is no significant trend in the proportion undergoing surgery $\left(\chi^{2}{ }_{1}=0.46, p=0.5\right)$. Trends in group characteristics and outcomes to June 2011 will be presented.
Conclusion Non-randomised evaluations of complex surgery are challenging but necessary. Ensuring the comparability of treatment groups is critical for robust evaluation.

\section{SP3-66 CARIES IN CHILDREN ON THE EAST SIDE OF MEXICO CITY STUDY}

doi:10.1136/jech.2011.1429760.66

L P S Martínez, * J M Núñez Martínez, C Osorno-Escareño Mdel, L Romero, U Érika Cenoz. Metropolitan Autonomous University Xochimilco, Distrito Federal, Mexico

Introduction The fluor addition in salt began in Mexico at the early '90s; consequently there has been a decrease of caries cases, but with different average results at the age of 12 in the whole country. Mexico City is one of the zones that present a moderate average (2.1-3). Considering this information it is pertinent to expose the benefit of fluoridated salt in different zones of Mexico City, as well as the different marginalisation characteristics.

Objective To know the experience of caries in children on the East side of Mexico City.

Methods Cross and observational survey. Informed previous consent, 552 students were analysed from the area of Iztapalapa on the East side of Mexico City. This zone is rated as one of the most marginalised ones of the federative entity. Previous calibration of two examiners, both caries dentitions were diagnosed (WHO). Data analysed with SPSS, getting central tendency results and dispersion of gender, age and caries variables. In order to distinguish homogeneity of variables it was used $\chi^{2}(p<0.05)$.

Results 285 girls (52\%) and 267 boys (48\%) were examined, average age of 8.65 years old. The global dmft was $3.79+3.48$ and the global DMFT was $1.67+2.07$. There was no significant gender difference in both dentitions, but there was one related to age $(p<0.0001)$. The average dmft- 6 was $4.98+4.10$ and the DMFT-12 was $3.22+2.71$.

Conclusions The studied population presented high rates of caries at the age of twelve, a situation that suggests major efforts in mouth health prevention

\section{SP3-67 KAPOSI SARCOMA INCIDENCE IN UGANDA AND ZIMBABWE, BEFORE AND DURING HIV/AIDS EPIDEMIC}

doi:10.1136/jech.2011.1429760.67

${ }^{1} \mathrm{~K}$ Chaabna, ${ }^{*} \mathrm{~F}$ Bray, ${ }^{2} \mathrm{H}$ Wabinga, ${ }^{3} \mathrm{E}$ Chokunonga, ${ }^{4,5} \mathrm{P}$ Vanhems, ${ }^{1} \mathrm{D}$ Forman ${ }^{1}$ International Agency for Research on Cancer, Lyon, France; ${ }^{2}$ Kampala Cancer registry, Department of Pathology, Makerere University, Kampala, Uganda; ${ }^{3} Z i m b a b w e ~ C a n c e r$ registry, Parirenyatwa Hospital, Harare, Zimbabwe; ${ }^{4}$ Service d'Hygiène, Epidémiologie et Prévention, Hôpital Edouard Herriot, Hospices Civils de Lyon, Lyon, France ${ }^{5}$ Laboratoire d'Epidémiologie et de Santé Publique, CNRS, UMR 5558, Université de Lyon, Université Lyon 1, Lyon, France

Introduction We described Kaposi sarcoma (KS) incidence changes during the AIDS emergence and subsequently in Zimbabwe with a high prevalence of HIV infection and Uganda with an intermediate HIV/AIDS prevalence and known to have high rates of endemic KS. Methods Cancer data were extracted from the cancer registries of Harare (1990-2005), Bulawayo (1963-1972), Zimbabwe and Kyadondo, Uganda (1960-1971 and 1991-2007). We used a join point model to analyse the time trends of age-standardised rates, and the populations were compared by computing the standardised incidence ratio and $95 \%$ CIs.

Results In both countries, an increase in the incidence of $\mathrm{KS}$ accompanied the AIDS emergence. In Harare, KS incidence seemed to change according to the time trend of HIV/AIDS prevalence; however, in Kyadondo, we observed an increase of KS incidence in people over 50 years despite the decrease in HIV prevalence. Before 
the AIDS epidemic, KS incidence in Bulawayo was similar to that in Kyadondo in both genders; however, in men it became higher in Harare than Kyadondo during the AIDS epidemic, thus seeming to follow the geographical distribution of HIV prevalence. In women, the geographical pattern of KS appeared independent of HIV prevalence. In the populations studied, despite HIV prevalence being higher in females than males, KS incidence was higher in males than females.

Conclusion HIV prevalence could have different impacts on KS incidence, possibly explained by other risk factor exposures. Furthermore, a higher risk of KS emergence in men or increased risk factor exposure in men could explain the gender differences.

\section{SP3-68 A SYSTEMATIC REVIEW OF INTERVENTIONS TO PREVENT OR REDUCE SUBSTANCE USE AND SEXUAL RISK BEHAVIOUR IN YOUNG PEOPLE}

doi:10.1136/jech.2011.1429760.68

C Jackson, ${ }^{*}$ R Geddes, S Haw, J Frank. Scottish Collaboration for Public Health Research and Policy, Edinburgh, UK

Background Interventions aimed at preventing risky behaviour in adolescence and young adulthood have largely focused on single risk behaviours and a limited number of underlying predictors. Interventions that take a broader approach to reducing risk behaviour may more effectively and efficiently reduce multiple risk behaviours. Methods We performed a systematic review to identify experimental studies of interventions to reduce risk behaviour in adolescents or young adults and that reported on any substance (alcohol, tobacco and illicit drug) use and sexual risk behaviour outcomes.

Results From 1129 articles, 18 experimental studies met our inclusion criteria, which were heterogeneous in nature and design. Study results were mixed, with programmes generally impacting on some outcome measures, but not others. The most promising interventions addressed multiple domains (individual and peer, family, school and community) of risk and protective factors for risk behaviour. There was some evidence that intervening in the early- to mid childhood school years has a high impact on later risk behaviour. Conclusions We found few studies that demonstrated a consistent, significant and sustained impact on risk behaviours. However, there is some evidence that programmes can have a long-term impact on multiple risk behaviours, with the most promising interventions addressing multiple domains of risk and protective factors. Complex interventions therefore need to be developed in the context of effective cross-sector engagement and collaboration. Furthermore, the current focus on investing in pre-school "early years" programmes should be extended to include the early- to mid-childhood school years.

\section{SP3-69 GENDER, ETHNIC AND FOOD INTAKE DIFFERENCES IN THE OCCURRENCE OF OVERWEIGHT IN INDIAN, NEPALESE AND SRILANKAN MEDICAL STUDENTS}

doi:10.1136/jech.2011.1429760.69

${ }^{1} \mathrm{~B}$ Sathian, ${ }^{*} \mathrm{R}$ Babu, ${ }^{2} \mathrm{~J}$ Sreedharan, ${ }^{1} \mathrm{~N}$ Bhat, ${ }^{1} \mathrm{~N}$ Chandrasekharan, ${ }^{3} \mathrm{E}$ Rajesh. ${ }^{1}$ Manipal College of Medical Sciences, Pokhara, Nepal; ${ }^{2}$ Gulf Medical University, Ajman, United Arab Emirates; ${ }^{3}$ MG University, Kerala, India

Introduction In developed and developing countries young people are at increased risk of obesity. Obesity is associated with chronic disease including Type II Diabetes, cardiovascular diseases and cancer. Body mass index (BMI) is commonly used to measure obesity. The aim of this study was to describe the prevalence of overweight among medical students in Nepal according to sex, ethnicity and diet (vegetarian/non-vegetarian).
Methods Approval for the study was obtained from the institutional research ethical committee. Participants were recruited from Manipal College of Medical Sciences, Pokhara, Nepal. BMI $\left(\mathrm{kg} / \mathrm{m}^{2}\right)$ was calculated using the height and weight. Data were analysed using Microsoft Excel (Windows 2003) and SPSS 16.

Results Of the 311 participants 179 (57.6\%) were Nepalese, 97 (31.2\%) Indian and 35 (11.2\%) Srilankan. $72 \%$ were males and $28 \%$ females. $80.1 \%$ were non-vegetarians and $19.9 \%$ were vegetarians. Overall $28.7 \%$ of subjects were overweight; $33 \%$ of Nepalese participants, $17.5 \%$ of Indian participants and $20 \%$ of Srilankans participants. $34.4 \%$ of men and $6.9 \%$ of women were overweight. $26.9 \%$ of non-vegetarian and $25.8 \%$ of vegetarian participants were overweight.

Conclusion In medical students in Nepal, gender, ethnicity and diet were associated with being overweight. Obesity prevention should focus on physical activity and diet.

\section{SP3-70 A NEW MODEL FOR ENVIRONMENTAL HEALTH DECISION MAKING AT A LOCAL LEVEL}

doi:10.1136/jech.2011.1429760.70

R Khan, ${ }^{*}$ D Lemon, D Phillips, E Youngman, R Partridge. NHS Dorset, Dorchester, Dorset, UK

Most environmental health issues are multi causal with complex non-linear relationships between the environment and health. New tools and methods are needed to assist policy and decision making that cut across traditional policy making structures and support collaboration by different agencies at different administrative and spatial scales. This project is aimed at developing a new environmental health decision making model to support integrated policy and action in environmental health, especially at a local level. We are piloting the model using both a setting based approach (Housing domain) and a health outcome approach (Chronic Obstructive Pulmonary disorder). The proposed decision making model is based on four stages: problem framing, environmental health intelligence, model building and appraisal. The problem framing stage involves conceptualising the problem using an ecological framework. The second stage involves developing environmental health indicators. The third stage involves converting the conceptual model into a simulation model using a system of differential equations and systems dynamics modelling. Appraisal represents the final stage in assessment, and provides the point at which results are synthesised and interpreted by the stakeholders. Results of the model will be presented at the conference. The aim of the decision making model is to enable stakeholders to understand the dynamics of environmental health issues and simulate and compare and evaluate different policy decisions and their financial and health impacts. This project will improve on current methods and tools to assess policy relevant problems that have implications for working across the environment and human health interface.

\section{SP3-71 QUALITY ASSESSMENT OF DIRECTLY OBSERVED TREATMENT OF TUBERCULOSIS IN DHAKA CITY}

doi:10.1136/jech.2011.142976o.71

${ }^{1} \mathrm{~S}$ Satter, ${ }^{*}{ }^{2} \mathrm{M}$ A Hafez, ${ }^{3} \mathrm{M}$ Flora, ${ }^{2} \mathrm{M}$ Shahjahan, ${ }^{2} \mathrm{~A}$ Afroz. ${ }^{1}$ International Center for Diarrhoeal Disease Research, Bangladesh (ICDDR,B), Dhaka, Bangladesh; ${ }^{2}$ Bangladesh Institute of Health Sciences (BIHS), Dhaka, Bangladesh; ${ }^{3}$ National Institute of Public Health \& Social Medicine (NIPSOM), Dhaka, Bangladesh

Introduction Quality of directly observed treatment (DOT) is key factors for successful TB control programme. This study was 